\section{InSTABILITY OF Nonlocal CONTINUUM aND Strain Averaging}

By Zdeněk P. Bažant, ${ }^{1}$ F. ASCE and Ta-Peng Chang, ${ }^{2}$ S. M. ASCE

Abstract: Nonlocal continuum, in which the (macroscopic smoothed-out) stres at a point is a function of a weighted average of (macroscopic smoothed-out) strains in the vicinity of the point, are of interest for modeling of heterogeneous materials, especially in finite element analysis. However, the choice of the weighting function is not entirely empirical but must satisfy two stability conditions for the elastic case: (1) No eigenstates of nonzero strain at zero stress, called unresisted deformation, may exist; and (2) the wave propagation speed must be real and positive if the material is elastic. It is shown that some weighting functions, including one used in the past, do not meet these conditions, and modifications to meet them are shown. Similar restrictions are deduced for discrete weighting functions for finite element analysis. For some cases, they are found to differ substantially from the restriction for the case of acontin if the averaging extends only over a few finite elements.

\section{INTRODUCTION}

Nonlocal continuum models, in which the stress at a given point is assumed to be a function of a weighted average of the strains within the neighborhood at that point, offer the possibility to take into account the stress-strain interaction at distance due to heterogeneity of the microstructure. Interest in this modeling approach was revived recently as it was realized that some sort of averaging over a characteristic volume is required to model the strain-softening zones in heterogeneous brittle materials and their progressive damage due to microcracking (1-3).

Some attempts have been made to apply nonlinear nonlocal material models in finite element analysis of dynamic failures caused by strainsoftening. Computer results, however, indicate that nonlocal material models are highly susceptible to various instabilities, not only in the strainsoftening range but also in the elastic range. The intent of this study is to examine the instabilities in the elastic range, which are entirely due to the modeling approach, in particular, the choice of the weighting function used for strain averaging.

\section{Nonlocal Medium}

In the classical, or local, theory of linear elasticity, the constitutive relation may be written as $\sigma_{i j}(\mathbf{x})=C_{i j k m}(\mathbf{x}) \epsilon_{k m}(\mathbf{x})$, in which $\sigma_{i j}, \epsilon_{i j}=$ Carte sian components of the stress and strain tensors; $C_{i j k m}=$ elastic constants that may depend on location vector; $\mathbf{x}$; and $\epsilon_{i j}=\left(u_{i, j}+u_{j, i}\right) / 2$, in which $u_{i}=$ displacement components (repeated subscripts imply summation

${ }^{1}$ Prof. of Civ. Engrg. and Dir., Center for Concrete and Geomaterials, Northwestern Univ., Evanston, Ill. 60201.

${ }^{2}$ Grad. Research Asst., Northwestern Univ., Evanston, Ill. 60201.

Note.-Discussion open until March 1, 1985. To extend the closing date one month, a written request must be filed with the ASCE Manager of Technical and Professional Publications. The manuscript for this paper was submitted for review and possible publication on January 27,1984. This paper is part of the Jourmal of Enginering Mechanics, Vol 110 No 10, October, 1984. OASCE, ISSN 0733-9399/84/0010-1441/\$01.00. Paper No. 19198. and subscripts preceded by a comma denote partial differentiation). The equations of motion are $\sigma_{i j, j}=\rho \ddot{u}_{i}$ in which $\rho$ is the mass density, and superior dots denote time derivatives.

In the theory of statistically inhomogeneous materials, it has been found that the smoothed-out macrostresses, $\sigma_{i j}$, and macrostrains, $\epsilon_{i j}$, representing certain averages of the actual randomly distributed microstresses and microstrains, do not have a point correlation. Rather, the entire (smoothed-out macroscopic) stress and strain fields within a certain characteristic volume are mutually related $(4,21-25)$. In the simplest theory of this kind, proposed by Kröner $(21,22)$, Kunin (24), Krumhansl (23), Beran and McCoy (4), and Levin (25), and developed in detail by Eringen and co-workers $(5-20)$, the components of the stress tensor, $\sigma_{i j}$ are assumed to be expressed by an averaging integral over the strains

$\boldsymbol{\sigma}_{i j}(\mathbf{x})=\int_{V} \alpha\left(\mathbf{x}-\mathbf{x}^{\prime}\right) C_{i j k m} \boldsymbol{\epsilon}_{k m}\left(\mathbf{x}^{\prime}\right) d V\left(\mathbf{x}^{\prime}\right) \ldots \ldots \ldots \ldots \ldots \ldots \ldots \ldots \ldots \ldots \ldots \ldots \ldots$

in which $V=$ volume of the body; $C_{i j k m}=$ the elastic moduli, which may, but need not, depend on $\mathbf{x}-\mathbf{x}^{\prime}$; the products of $\alpha\left(x-\mathbf{x}^{\prime}\right) C_{i j k m}$ are called the nonlocal elastic moduli; and $\alpha\left(x-x^{\prime}\right)$ is a given weighting function of the distance $\left|\mathbf{x}-\mathbf{x}^{\prime}\right|$. This function must satisfy the normalizing condition, $\int_{V} \boldsymbol{\alpha}\left(\mathbf{x}-\mathbf{x}^{\prime}\right) d V=1$, so that the correct constitutive relation is obtained for the special case of uniform strain $(\epsilon=$ const.). The differential equation of motion has generally been considered in a the usual form, $\sigma_{i j, j}=\rho \ddot{u}_{, i}$. Recently it has been suggested that it is also possible to introduce an averaging integral on the right-hand side of the equation of motion, i.e. over the term $\rho \ddot{u}_{, i}$. Such generalizations, however, are not considered in this paper.

TABLE 1.-Fourier Transforms of Various Weighting Functions

\begin{tabular}{|c|c|c|c|c|c|}
\hline $\begin{array}{l}\text { Number } \\
\text { (1) }\end{array}$ & $\begin{array}{l}\text { Name } \\
\text { (2) }\end{array}$ & $\begin{array}{l}\text { Weighting function, } \alpha(s) \\
\text { (3) }\end{array}$ & $\begin{array}{l}\text { Interval } \\
\text { (4) }\end{array}$ & $\begin{array}{l}\text { Fourier transform, } \alpha^{*}(\omega) \\
\text { (5) }\end{array}$ & $\begin{array}{l}\text { Sign } \\
\text { (6) }\end{array}$ \\
\hline 1 & $\begin{array}{l}\text { Normal } \\
\text { distri- } \\
\text { bution }\end{array}$ & $\frac{1}{\sqrt{2 \pi} l} e^{-s^{2} / 2 l^{2}}$ & $-x<s<x$ & $e^{-\omega \omega_{i}^{2} / 2}$ & $>0$ \\
\hline 2 & Triangular & $\frac{2}{l}\left(1-\frac{2}{l}|s|\right)$ & $|s|<\frac{1}{2}$ & $2\left(\frac{2}{\omega l}\right)^{2}\left(1-\cos \frac{\omega l}{2}\right)$ & $\geq 0$ \\
\hline 3 & Uniform & & $|s|<\frac{l}{2}$ & $\frac{2}{\omega l} \sin \frac{\omega l}{2}$ & $\$ 0$ \\
\hline 4 & Spike & $\delta(s)$ & $-\infty<s<\infty$ & 1 & $>0$ \\
\hline 5 & $\begin{array}{l}\text { Spiked } \\
\text { uniform }\end{array}$ & $c \delta(s)+\frac{1-c}{l}$ & $|s|<\frac{1}{2}$ & $c+(1-c) \frac{2}{\omega l} \sin \frac{\omega l}{2}$ & \\
\hline 6 & $\begin{array}{l}\text { Spiked } \\
\text { trian- } \\
\text { gular }\end{array}$ & $c \delta(s)+(1-c) \frac{2}{l}\left[1-\frac{2}{l}|s|\right]$ & $|s|<\frac{l}{2}$ & $c+2(1-c)\left(\frac{2}{\omega l}\right)^{2}\left(1-\cos \frac{\omega l}{2}\right)$ & \\
\hline 7 & $\begin{array}{l}\text { Bilateral } \\
\text { expon- } \\
\text { ential }\end{array}$ & $\frac{1}{2 l} e^{-t s / 1}$ & $-\infty<s<\infty$ & $\frac{1}{1+(\omega l)^{2}}$ & $>0$ \\
\hline 8 & $\begin{array}{l}\text { Cauchy } \\
\text { distri- } \\
\text { bution }\end{array}$ & $\frac{1}{\pi} \frac{l}{l^{2}+s^{2}}$ & $-\infty<s<\infty$ & $e^{-(\omega)^{\prime}}$ & $>0$ \\
\hline 9 & $\begin{array}{l}\text { Hyperbolic } \\
\text { cosine }\end{array}$ & {$\left[\pi l \cosh \left(\frac{s}{l}\right)\right]^{-1}$} & $-\infty<s<\infty$ & {$\left[\cosh \left(\frac{\pi \omega l}{2}\right)\right]^{-1}$} & $>0$ \\
\hline
\end{tabular}



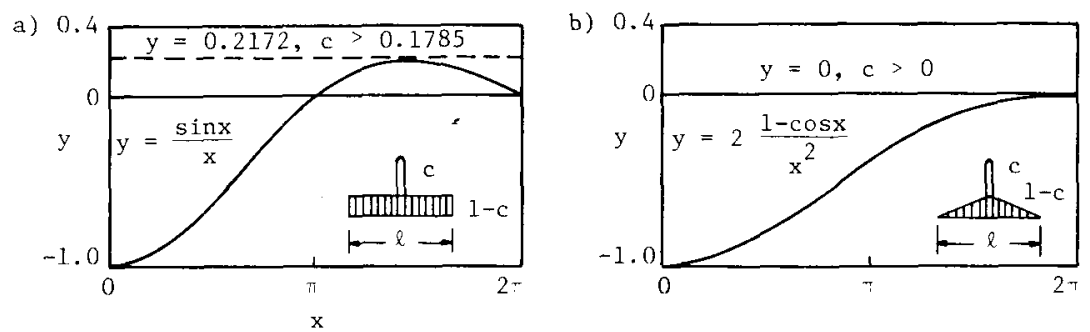

FIG. 1.-Minimum Value for Spike Coefficient, $c$

For the sake of simplicity, we consider only an infinite elastic body subjected to one-dimensional deformation, for which Eq. 1 may be simplified as

$\sigma(x)=E\langle\epsilon(x)\rangle, \quad\langle\epsilon(x)\rangle=E \int_{-\infty}^{\infty} \epsilon^{\prime}(x+s) \alpha(s) d s \ldots \ldots \ldots \ldots \ldots \ldots \ldots$

with $\int_{-\infty}^{\infty} \alpha(s) d s=1$

in which $s=x^{\prime}-x ; \sigma=$ stress; $\epsilon=\partial u / \partial x=$ strain $(\sigma$ and $\epsilon$ are both smoothed-out macroscopic quantities); $\langle\epsilon\rangle=$ average strain; $u=$ displacement; $E=$ Young's modulus, which is assumed to be constant; and $\alpha(s)$ $=$ a given empirical weighting function, which represents a material property and is symmetric, i.e., $\alpha(-s)=\alpha(s)$. Table 1 lists various possible simple formulas for $\alpha(s)$, of which the first two have been used by Eringen and co-workers (5-20). In these expressions, $l$ represents a characteristic averaging length (Fig. 1), which is a property of the given material and may be regarded as having a certain fixed ratio to the maximum size of the inhomogeneities in the microstructure. The normalizing condition (Eq. 3) ensues from the requirement that, for a homogeneous strain state $(\epsilon=$ const.), Eq. 2 must reduce to $\sigma(x)=E \epsilon(x)$

The classical, local continuum is obtained as a special case when $\alpha(s)$ $=\delta(s)=$ Dirac's delta function (No. 4 in Table 1). As will be seen later there is some merit in considering various linear combinations of a local continuum and a nonlocal continuum. Such combinations may be obtained by superimposing a spike on a smooth distribution, as exemplified in Table 1, Nos. 4 and 5 , in which $c$ is an arbitrary real coefficient, $0 \leq c \leq 1$. For $c=0$, we have the usual nonlocal medium (No. 3 or 2), and for $c=1$, we have the classical local medium as a special case (No. 4).

\section{Unresisted Deformation and Wave Propagation}

Any theory of an elastic continuum must obviously satisfy the follow ing two requirements:

Requirement I.- If the stresses, $\sigma(x)$, are everywhere zero, the strains in a stable material must be also zero, i.e., no unresisted deformation (zero-energy deformation mode) may be permitted by the theory.
Requirement II.--In a stable material, the wave propagation velocity, $v$, must be real.

From these two requirements it follows that the Fourier transform of the weighting function, $\alpha(s)$, must be positive for all real $\omega$, i.e.

$\alpha^{*}(\omega)=\int_{-\infty}^{\infty} e^{-i \omega s} \alpha(s) d s>0$

Let us now prove this condition, considering Requirement I first. According to Eq. 2, $\sigma(x)=0$ occurs when

$\int_{-\infty}^{\infty} \epsilon(x+s) \alpha(s) d s=0$

This condition may not have any nonzero solution (eigenstate). A general strain distribution may be approximated as $\epsilon(x)=\sum_{k} a_{k} \exp \left(i \omega_{k} x\right)$, in which $a_{k}$ and $\omega_{k}$ are some real numbers $(k=1,2,3, \ldots)$, and the actual strain is to be understood as the real part. No single term of this expansion, i.e.

$\epsilon(x)=a e^{i \omega x}$

with a real amplitude, $a$, and a real frequency, $\omega$, may satisfy Eq. 5 . Substituting Eq. 6 into Eq. 5, we obtain the condition that the equation $\int_{-\infty}^{\infty} \alpha(s) \exp [i \omega(x+s)] d s=0$ must not have any solution, and dividing this equation by $\exp (i \omega x)$, we conclude that $\alpha^{*}(\omega)$ must not be zero for any $\omega$. Since $\alpha^{*}(\omega)$ must be continuous, it must be either positive everywhere or negative everywhere. That a positive $\alpha^{*}(s)$ is the only possibility cannot be proven without Requirement II.

Second, consider the Requirement II. We restrict attention to small deformations, such that in one dimension $\epsilon(x)=\partial u(x) / \partial x$, in which $u$ $=$ displacement. The equation of motion is $\partial \sigma / \partial x=\rho \partial^{2} u / \partial t^{2}$, in which $t=$ time and $\rho=$ mass density. Eq. 2 then yields

$E \frac{\partial}{\partial x} \int_{-\infty}^{\infty} \frac{\partial u(z)}{\partial z} \alpha(x-z) d z=\rho \frac{\partial^{2} u}{\partial t^{2}}$

$(z=x+s)$

Any wave may be decomposed into harmonic components of the type $u(x)=a e^{i \omega(x-v t)}$

in which $v=$ wave velocity, $\omega ; a=$ real constants; and $\omega \neq 0$ (since for $\omega=0$ there is no strain). Substituting Eq. 8 into Eq. 7, multiplying the equation by $[\exp (i \omega v t)] /(i \omega \rho a)$, and substituting $z=x+s, d z=d s$, we obtain

$\frac{E}{\rho} \frac{\partial}{\partial x}\left[e^{i \omega x} \int_{-\infty}^{x} e^{i \omega s} \alpha(-s) d s\right]=v^{2} i \omega e^{i \omega x}$

Furthermore, substituting $s=-y$, and noting that $\alpha(-s)=\alpha(s)$, we find that the integral in this equation equals $\int_{-\infty}^{\infty} \exp (-i \omega y) \alpha(y) d y$, which represents $\alpha^{*}(\omega)$. Then, differentiating $e^{i \omega x} \alpha^{*}(\omega)$ with respect to $x$, and dividing the equation by $i \omega e^{i \omega x}$, we finally get $v^{2}=\frac{E}{\rho} \alpha^{*}(\omega)$ 
We see that $v$ is real if and only if $\alpha^{*}(\omega)$ is positive for all $\omega$ except $\omega=$ 0 . The fact that $\alpha^{*}(\omega)$ must be positive also for $\omega=0$ follows from Requirement I, as already proven. For the usual local continuum (Table 1 No. 4), we have $\alpha^{*}(\omega)=1$ for all $\omega$.

Note that Requirement $I$ is applied only to an elastic continuum. It could not be applied, of course, to inelastic materials with residual stresses. However, Requirement I could then be replaced, with equal results, by the requirement that, if the stress rate, $\dot{\sigma}$, is zero, the strain rate $\dot{\epsilon}$ must also be zero, provided that the tangent modulus, $E_{\mathrm{t}}=d \sigma / d \epsilon$, is positive.

\section{Examination of Some Weighting Functions}

Eq. 10 with the condition $\alpha^{*}(\omega)>0$ was obtained in a somewhat different manner by Eringen (Ref. 5, Eq. 4.11) as part of a wave dispersion study. It seems, however, that Eq. 10 has not yet been used to check various weighting functions, $\alpha(s)$, and, in fact, one function that violates the condition $\alpha^{*}(\omega)>0$ has been used subsequently.

Looking now at Table 1 , we see that the condition $\alpha^{*}(\omega)>0$ is not satisfied for the triangular weighting function, used previously in some works $(12,14,16,19)$. In finite element analysis, it is found that, when this function is used, spurious oscillations rapidly develop and soon produce an overflow number.

Further, we may note that the uniform weighting function (Table 1, No. 3) is also inadmissible. This simple function has, however, one important advantage for the modeling of inelastic behavior. For this function, and only for it, $l\langle\epsilon(x)\rangle$ represents exactly the change of distance between material points at $x+l / 2$ and $x-l / 2$, which appears to be useful for models of strain-softening (2).

Correction for the uniform weighting function (No. 3) or the triangular one (No. 2) may be obtained by using a combination of local and nonlocal media. Such a combination, which has already been considered by Eringen (18) for the purpose of matching (by a nonlocal continuum) the exact wave dispersion relation for an atomic lattice, may be obtained by superimposing a spike in the form of Dirac delta function, as indicated in Table 1, Nos. 5 and 6. For the spiked triangular distribution, its Fourier transform (No. 6 in Table 1) is always positive if $c>0$. Thus, it suf fices to choose any coefficient $c>0$ to prevent nonzero stress states at a zero strain state.

For the spiked uniform distribution we need, according to Table 1, No. 5 , that

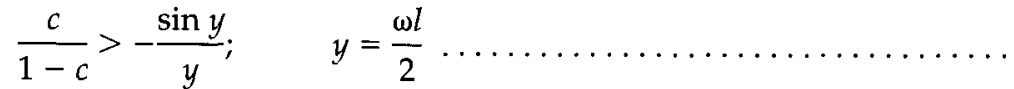

The location of the highest maximum of the right-hand side for $y>$ 0 is given by the condition $\tan y=y$, from which $y=4.49341$. Eq. 9 then yields the condition

$$
\text { c }>0.178465 \text { (Table 1, No. 5) }
$$

\section{Finite Element Approximations}

When the continuum is approximated by finite elements, the stability conditions may be different. Are they more severe or less severe?
Consider a one-dimensional system of identical constant-strain elements (Fig. 2), with nodes numbered as $k=1,2,3, \ldots$ Noting that the strain in the $k$ th element is $\epsilon_{k}=\left(u_{k+1}-u_{k}\right) / h$, in which $u_{k}$ are the nodal displacements and $h$ is the element length, Eq. 2 for the average stress may be approximated as

$\sigma_{k}=E\left[\frac{c}{h}\left(u_{k+1}-u_{k}\right)+\frac{1-c}{h} \sum_{j=-n}^{n} \alpha_{j}^{\prime}\left(u_{k+j+1}-u_{k+j}\right)\right]$

in which $\sigma_{k}$ denotes the average stress in the $k$ th element bordered by nodes $k$ and $k+1$; subscripts $j, k$ refer to element or node numbers; $n$ is the number of elements over which the averaging is made at each side of the central element; $c=$ coefficient of a superimposed delta function spike; and $\alpha_{j}^{\prime}$ are the given weights for the case without the spike. These weights may be chosen either as the values of $\alpha(z)$ at element centroids, or (better) as the integrals of $\alpha(z)$ over the areas of the respective elements, with $s$ being measured from the centroid of the element number, $k$. These weights must be normalized so that $\Sigma \alpha_{j}^{\prime}=1$.

According to Requirement $\mathrm{I}$, the difference equation in Eq. 13 may not allow a nonzero solution (eigenstate) when $\sigma_{k}=0$ for all $k$. We seek a solution of the form

$u_{k}=A e^{i \omega h k} \quad(k=1,2, \ldots)$

in which $A$ and $\omega$ are real constants, and $i^{2}=-1$. Substituting this into Eq. 13 where $\sigma_{k}=0$, we get
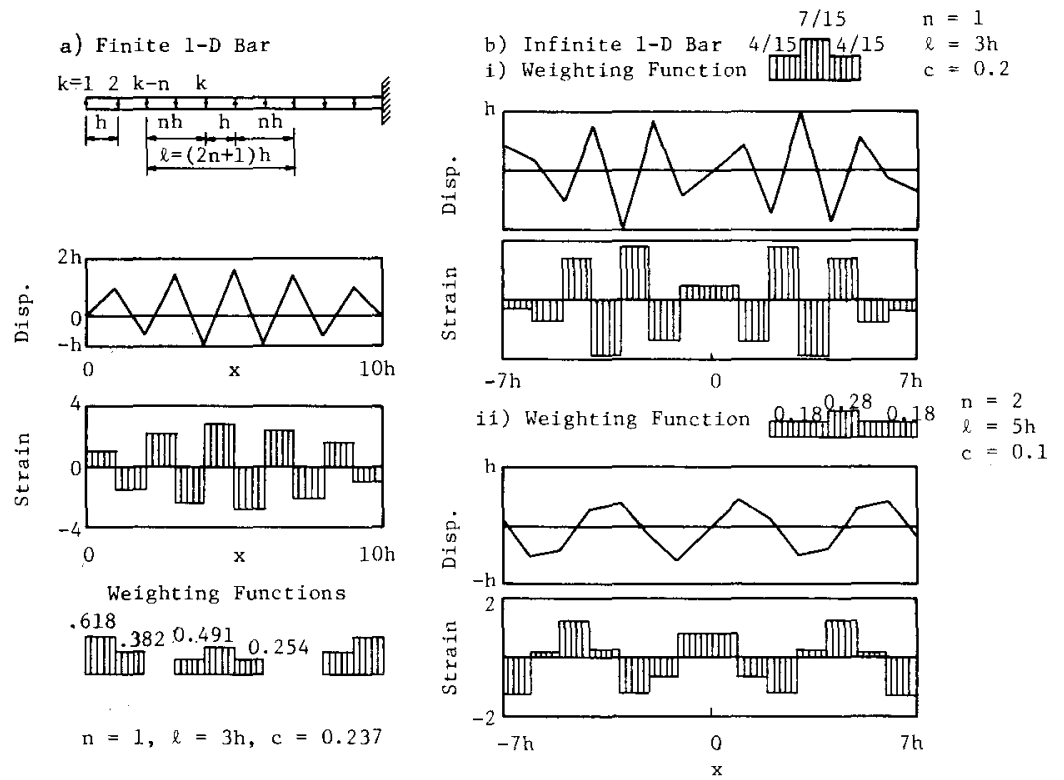

FIG. 2.-Nonzero Strain Fields at Zero Stress 
TABLE 2.-Minimum Values of Spike Coefflcient, $c$, for Finite Element System and Uniform Weights

\begin{tabular}{c|c|c|c|c|c|c|c}
\hline \hline $\begin{array}{c}n \\
(1)\end{array}$ & $\begin{array}{c}c \\
(2)\end{array}$ & $\begin{array}{c}n \\
(3)\end{array}$ & $\begin{array}{c}c \\
(4)\end{array}$ & $\begin{array}{c}n \\
(5)\end{array}$ & $\begin{array}{c}c \\
(6)\end{array}$ & $\begin{array}{c}n \\
(7)\end{array}$ & $\begin{array}{c}c \\
(8)\end{array}$ \\
\hline 1 & 0.25000 & 4 & 0.18472 & 8 & 0.18019 & 30 & 0.17860 \\
2 & 0.20000 & 5 & 0.18261 & 10 & 0.17959 & 50 & 0.17851 \\
3 & 0.18898 & 6 & 0.18142 & 20 & 0.17876 & $\infty$ & 0.17847 \\
\hline \hline
\end{tabular}
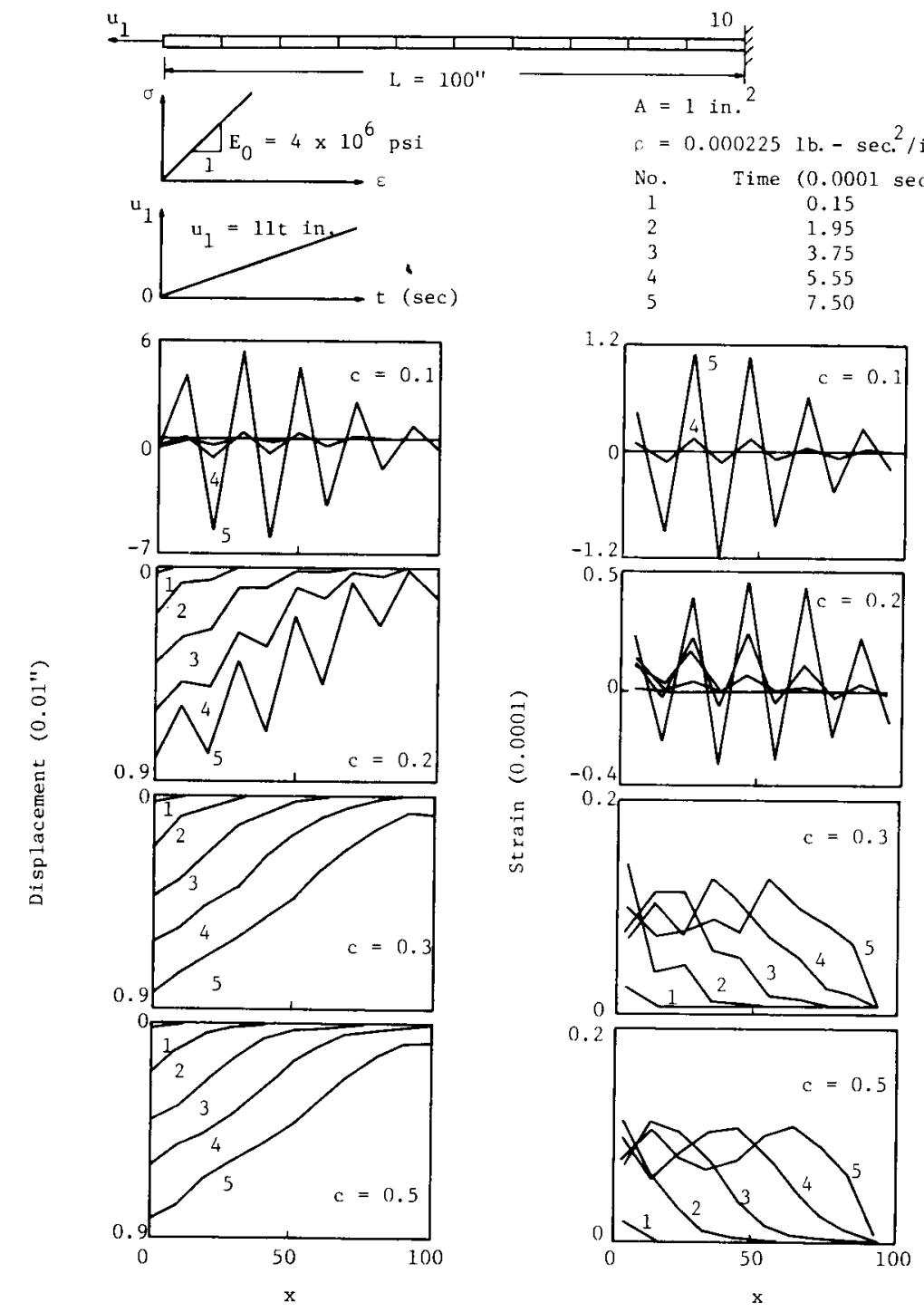

FIG. 3.-Displacement and Strain Fields for Spiked Uniform Weighting Function $c\left(e^{i \omega h}-1\right)+(1-c) \sum_{j=-n}^{n} \alpha_{j}^{\prime}\left(e^{i(j+1) \omega h}-e^{i j \omega h}\right)=0$

The real part of this equation yields

$c=\left(1-\frac{\sin y}{\sum_{j=-n}^{n} \alpha_{j}^{\prime} \sin [(2 j+1) y]}\right)^{-1} ; \quad y=\frac{\omega h}{2} \ldots$

and the imaginary part is found to yield the same equation.

Now, to avoid unresisted deformations, the value of $c$ must be larger than the largest value of the right-hand side function in Eq. 16 for all real $\omega$. Since this function is periodic with period $4 \pi$, one needs to look for the maximum only within the interval $0 \leq \omega \leq 4 \pi$. The maximums have been found by computer search first for the uniform distribution, $\alpha_{j}^{\prime}=1 / l$, and the results are summarized in Table $2 ; c$ must be larger than the values in this Table.

The maximums of the right-hand side of Eq. 16 have then been found for $\alpha_{j}^{\prime}$ according to the triangular distribution, the normal distribution, and the bilateral exponential distribution. For these distributions, the maximum right-hand side of Eq. 16 was found to be always negative. So we conclude that the discrete forms of these distributions are always admissible even without the spike. This result is interesting for the triangular distribution since its continuous form (without a spike) is not admissible. Here, the discretization makes the limit on $c$ less severe than for the continuum version, whereas for the uniform distribution, the discretization makes the limit on $c$ more severe (Table 2).

Experience with numerical finite element calculations shows that the solution does not behave well if $c$ is close to its minimum admissible value. The situation seems similar to that in solving a linear equation system whose matrix is nearly singular (ill-conditioned). Noise in the form of large oscillations builds up if $c$ is close to its limiting value. Therefore, the difference of $c$ from its limiting value must be finite and sufficiently large. Numerical experience showed that $c>0.3$ is required for the uniform weighting function for a continuum (Fig. 3).

\section{Conclusions}

1. In the formulation of nonlocal elasticity, the weighting function used for strain averaging must be chosen such that: (1) Unresisted deformations, i.e., deformations at zero stress, cannot take place; and (2) the wave propagation speeds must be real.

2. Not all reasonable looking weighting functions, and not all of those used in the past, satisfy this condition. One of those is the triangular weighting function. Remedy can be obtained by superimposing a delta function spike on the weighting function, which is equivalent to a linear combination of stresses from local and nonlocal continua.

3. In finite element applications, the conditions resulting from the foregoing requirements are similar but not exactly the same. The use of 
a weighting function that is close to violating the foregoing requirements causes numerically difficulties.

\section{ACKNOWLedgment}

Financial support under AFOSR Grant No. 83-0009 to Northwestern University is gratefully acknowledged. Thanks are also due to Professor Ted. B. Belytschko for various stimulating discussions.

\section{Appendix.-References}

1. Bažant, Z. P., "Crack Bank Model for Fracture of Geomaterials," Proceeding of the Fourth International Conference on Numerical Methods in Geomechanics, held at the University of Alberta, Z. Eisenstein, ed., Edmonton, Vol. 3, 1982, pp $1137-1162$.

2. Bažant, Z. P., Belytschko, T. B., and Chang, T. P., "Continuum Model for Strain Softening," Report No. 83-11/401c, Center for Concrete and Geomaterials, Northwestern University, Evanston, Ill., Nov., 1983.

3. Bažant, Z. P., and Oh, B. H., "Crack Band Theory for Fracture of Concrete," Materials and Structures, RILEM, Paris, France, Vol. 16, 1983, pp. 155-177

4. Beran, M. J., and McCoy, J. J., "Mean Field Variations in a Statistical Sample of Heterogeneous Linearly Elastic Solids," International Journal of Solids Structures, Vol. 6, 1970, pp. 1035-1054.

5. Eringen, A. C., "Nonlinear Polar Elastic Continua," International Journal of Engineering Science, Vol. 10, 1972, pp. 1-16.

6. Eringen, A. C. "Linear Theory of Nonlocal Elasticity and Dispersion of Plane Waves," International Journal of Engineering Science, Vol. 10, 1972, pp. 425435.

7. Eringen, A. C., "On Rayleigh Surface Waves With Small Wave Lengths," Letters in Applied and Engineering Sciences, Vol. 1, 1973, pp. 11-17.

8. Eringen, A. C., "Theory of Nonlocal Thermoelasticity, International Journal of Engineering Science, Vol. 12, 1974, pp. 1063-1077.

9. Eringen, A. C., "Continuum Mechanics at the Atomic Scale," Crystal Lattice Defects, Vol. 7, pp. 109-130.

10. Eringen, A. C.. "Edge Dislocation in Nonlocal Elasticity," International Journal of Engineering Science, Vol. 15, 1977, pp. 177-183.

11. Eringen, A. C., "Screw Dislocation in Non-local Elasticity," Journal of Physics D: Applied Physics, Vol. 10, 1977, pp. 671-678

12. Eringen, A. C., "Nonlocal Continuum Mechanics and Some Applications," Lectures given at NATO Advanced Study Institute on Nonlinear Equation in Physics and Mathematics, Istanbul, Turkey, Aug., 1977, pp. 271-318.

13. Eringen, A. C., "Line Crack Subject to Antiplane Shear," Engineering Fracture Mechanics, Vol. 12, 1979, pp. 211-219.

14. Eringen, A. C. "Linear Crack Subject to Shear," International Journal of Fracture, Vol. 14, No. 4, Aug., 1978.

15. Eringen, A. C., and Ari, N., "Nonlocal Stress Field at Griffith Crack," Crystal Lattice Defects and Amorphous Materials, Vol. 10, 1983, pp. 33-38.

16. Eringen, A. C., and Balta, F., "Screw Dislocation in Non-local Hexagonal Elastic Crystals," Crystal Lattice Defects, Vol. 7, 1978, pp. 183-189.

17. Eringen, A. C., and Balta, F., "Penetration of a Half Space by a Rectangular Cylinder," Journal of Applied Mechanics, Vol, 46, No. 3, Sept., 1979.

18. Eringen, A. C. and Edelen, D. G. B. "On Nonlocal Elasticity," International Journal of Engineering Science, Vol. 10, 1972, pp. 233-248.

19. Eringen, A. C., and Kim, B. S., "Relation Between Nonlocal Elasticity and Lattice Dynamics," Crystal Lattice Defects, Vol. 7, 1977, pp. 51-57.

20. Eringen, A. C., Speziale, C. G., and Kim, B. S., "Crack-Tip Problem in Nonlocal Elasticity," Journal of Mechanics and Physics of Solids, Vol. 25, 1977, pp 339-355.
21. Kröner E "Elasticity Theory of Materials with Long Range Cohesive Forces," International Journal of Solids Structures, Vol. 3, 1967, pp. 731-742.

22. Kröner E "Interrelations Between Various Branches of Continuum $\mathrm{Me}$ chanics," Mechanics of Generalized Continua, E. Kröner, ed., Springer-Verlag Heidelberg, Germany, 1968, pp. 330-340.

23. Krumhansl, J. A., "Some Considerations of the Relation Between Solid State Physics and Generalized Continuum Mechanics," Mechanics of Generalized Continua, E. Kröner, ed., Springer-Verlag, Heidelberg, Germany, 1968, pp 298-311.

24. Kunin I A "The Theory of Elastic Media With Microstructure and the Theory of Dislocations," Mechanics of Generalized Continua, E. Kröner, ed., Springer-Verlag, Heidelberg, Germany, 1968, pp. 321-328.

25. Levin, V. M., "The Relation Between Mathematical Expectations of Stress and Strain Tensors in Elastic Microheterogeneous Media," Journal of Applied Mathematics and Mechanics, translation of Prikladnaya Matematika $i$ Mekhanika, Vol. 35, No. 4, 1971, pp. 694-701. 